International Journal of Pure and Applied Mathematics

Volume 85 No. 4 2013, 751-759

ISSN: 1311-8080 (printed version); ISSN: 1314-3395 (on-line version)

url: http://www.ijpam.eu

doi: http://dx.doi.org/10.12732/ijpam.v85i4.11

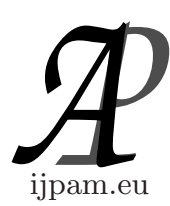

\title{
CODES SATISFYING THE CHAIN CONDITION WITH POSET WEIGHTS
}

\author{
Luciano Panek \\ Center of Exact Sciences and Engineering \\ State University of West Parana \\ 85870-650, Foz do Iguaçu, PR, BRAZIL
}

\begin{abstract}
In this paper we extend the concept of generalized Hamming weights for poset-weight codes and show that any linear code $C$ satisfies the chain condition if support of $C$ is a totally ordered subposet.
\end{abstract}

AMS Subject Classification: 94B05, 06A06

Key Words: poset codes, generalized Hamming weights, chain condition, total order

\section{Introduction}

In 1995, Brualdi, Graves and Lawrence ([1]) extended the scope of metrics to be considered in coding theory, considering the notion of poset-codes, as we briefly introduce in the next paragraph.

Let $(P, \leq)$ be a partially ordered finite set, abbreviated as poset, and assume $P=\{1,2, \ldots, n\}$. An ideal $I$ of $P$ is a subset of $P$ with the property that $y \in I$ and $x \leq y$, implies that $x \in I$. Given $A \subset P$, we denote by $\langle A\rangle$ the smallest ideal of $P$ containing $A$. Given $x=\left(x_{1}, \ldots, x_{n}\right) \in \mathbb{F}_{q}^{n}$, the support of $x$ is the set

$$
\operatorname{supp}(x):=\left\{i \in P: x_{i} \neq 0\right\} .
$$

We define the poset-weight $w_{P}$ of $x$ (also called $P$-weight), as the cardinality of the smallest ideal containing $\operatorname{supp}(x)$, that is

Received: February 21, 2013

(C) 2013 Academic Publications, Ltd. url: www.acadpubl.eu 


$$
w_{P}(x):=|\langle\operatorname{supp}(x)\rangle|
$$

The $P$-weight $w_{P}$ induces a metric in the vector space $\mathbb{F}_{q}^{n}$ defined by $d_{P}(x, y)=$ $w_{P}(x-y)([1$, Lemma 1.1]). If $P$ is antilinear, i.e., $x \leq y$ iff $x=y$, then the $P$ weight is the usual Hamming weight $w_{H}$. An important family of poset weights (non-Hamming weights) which can be applied to concrete communication systems are Rosenbloom-Tsfasman weights (see [6], [5]), and a particular case of interest is a linear (or total) order: $i_{1}<i_{2}<\ldots<i_{n}$.

Motivated by several applications in cryptography, Wei introduced in 1991 the concept of generalized Hamming weights ([7]). We extend here the concept of generalized Hamming weights to poset-weights. Let $P=\{1,2, \ldots, n\}$ be a partially ordered set. If $D$ is a linear subspace of the linear code $C$ we write $D \leq C$. When $D$ is a proper subspace of $C$ we write $D<C$. The generalized $P$-weight $\|\cdot\|_{P}$ of a $r$-dimensional subspace $D \leq \mathbb{F}_{q}^{n}$ is defined as

$$
\|D\|_{P}=\left|\bigcup_{x \in D}\langle\operatorname{supp}(x)\rangle\right| .
$$

The $r$-th $P$-weight of a $k$-dimensional code $C \leq \mathbb{F}_{q}^{n}$ is

$$
d_{(P, r)}(C)=\min \left\{\|D\|_{P}: D \leq C, \operatorname{dim}(D)=r\right\} .
$$

Since it will cause no ambiguity, we denote $d_{(P, r)}(c)$ simply by $d_{r}(C)$. A $k$ dimensional code $C \leq \mathbb{F}_{q}^{n}$ with $P$-weights hierarchy $\left(d_{1}(C), \ldots, d_{k}(C)\right)$ is called an $\left[n ; k ; d_{1}(C), \ldots, d_{k}(C)\right]_{q}$-code.

Many new perfect codes have been found with such poset-metrics (see [2], for example). Motivated by this fact we investigated in this work the possibility of the existence of new codes satisfying the chain condition with the generalized $P$-weights. In the terminology of Wei and Yang ([8]), a $k$-dimensional code $C \leq \mathbb{F}_{q}^{n}$ satisfies the chain condition if there exists a sequence of nested linear subspaces (maximal flag)

$$
D_{1}<D_{2}<\ldots<D_{k-1}<D_{k}=C
$$

with $\left\|D_{r}\right\|_{P}=d_{r}(C)$ and $\operatorname{dim}\left(D_{r}\right)=r$ for every $r \in\{1,2, \ldots, k\}$. If $P$ is antilinear $\left(w_{P}=w_{H}\right)$ the Hamming codes, dual Hamming codes, Reed-Muller codes for all orders, maximum-separable-distance codes and Golay codes satisfy the chain condition (see [8]). Moreover, every perfect code must be a code satisfying the chain condition (see [3]). 
In this work we will show that any poset-code $C \leq \mathbb{F}_{q}^{n}$ with support totally ordered satisfies the chain condition. Moreover, the sequence of linear subspaces $D_{1}<D_{2}<\ldots<D_{k-1}<D_{k}=C$ that achieve the $P$-weights is unique. It will follow that if $\left\|D_{r}\right\|_{P}=d_{r}(C)$ with $D_{r} \leq C$ for every $r \in\{1,2, \ldots, k\}$, then $D_{1}<D_{2}<\ldots<D_{k-1}<D_{k}=C$.

\section{Codes Satisfying the Chain Condition}

Before we show that any poset-code with support totally ordered satisfies the chain condition, we give an example in the case that $P$ is a weak order and shows the monotonicity of the minimum poset-weights.

We denote by span $X$ the linear subspace of $\mathbb{F}_{q}^{n}$ spanned by the set $X \subset \mathbb{F}_{q}^{n}$.

Example 2.1. Let $P=n_{1} \mathbf{1} \oplus \ldots \oplus n_{9} \mathbf{1}$ be the weak order given by the ordinal sum of the antilinear subposets $n_{1} \mathbf{1}, \ldots, n_{9} \mathbf{1}$ with 3 elements. Explicitly, $P$ is the poset whose underlying set is

$$
\{1,2, \ldots, 27\}=n_{1} \mathbf{1} \cup \ldots \cup n_{9} \mathbf{1}
$$

with

$$
n_{1} \mathbf{1}=\{1,2,3\}, n_{2} \mathbf{1}=\{4,5,6\}, \ldots, n_{9} \mathbf{1}=\{25,26,27\}
$$

and order relation are given by $x<y$ if and only if $x \in n_{i} \mathbf{1}, y \in n_{j} \mathbf{1}$ for some $i, j$ with $i<j$.

If $M_{9 \times 3}\left(\mathbb{F}_{2}\right)$ is the linear space of all $9 \times 3$ matrices over the finite field $\mathbb{F}_{2}$, we defined the poset-weight $w_{P}$ of

$$
x=\left(\begin{array}{ccc}
a_{1} & a_{2} & a_{3} \\
a_{4} & a_{5} & a_{6} \\
a_{7} & a_{8} & a_{9} \\
\vdots & \vdots & \vdots \\
a_{22} & a_{23} & a_{24} \\
a_{25} & a_{26} & a_{27}
\end{array}\right) \in M_{9 \times 3}\left(\mathbb{F}_{2}\right)
$$

as $w_{W}(x)=w_{W}\left(a_{1}, a_{2}, a_{3}, \ldots, a_{25}, a_{26}, a_{27}\right)$. 
Consider the $[27 ; 3]_{2}$ code $C$ spanned by $\left\{v_{1}, v_{2}, v_{3}\right\}$ where

$$
v_{1}=\left(\begin{array}{lll}
1 & 0 & 0 \\
1 & 0 & 0 \\
1 & 0 & 0 \\
0 & 0 & 0 \\
0 & 0 & 0 \\
0 & 0 & 0 \\
0 & 0 & 0 \\
0 & 0 & 0 \\
0 & 0 & 0
\end{array}\right), v_{2}=\left(\begin{array}{lll}
0 & 0 & 0 \\
0 & 0 & 0 \\
0 & 0 & 0 \\
0 & 1 & 0 \\
0 & 1 & 0 \\
0 & 1 & 0 \\
0 & 0 & 1 \\
0 & 0 & 0 \\
0 & 0 & 0
\end{array}\right), v_{3}=\left(\begin{array}{lll}
0 & 0 & 0 \\
0 & 0 & 0 \\
0 & 0 & 0 \\
0 & 0 & 0 \\
0 & 0 & 0 \\
0 & 1 & 0 \\
0 & 0 & 1 \\
0 & 0 & 1 \\
0 & 0 & 1
\end{array}\right)
$$

Considering the usual Hamming weight $w_{H}, C$ is a $[27 ; 3 ; 3,6,9]_{2}$-code that does not satisfy the chain condition, since the weight hierarchy is achieved by the subspaces $\operatorname{span}\left\{v_{1}\right\}, \operatorname{span}\left\{v_{2}, v_{3}\right\}, \operatorname{span}\left\{v_{1}, v_{2}, v_{3}\right\}=C$ :

$$
\begin{aligned}
& d_{(H, 1)}=3=\left\|\operatorname{span}\left\{v_{1}\right\}\right\| \\
& d_{(H, 2)}=6=\left\|\operatorname{span}\left\{v_{2}, v_{3}\right\}\right\| \\
& d_{(H, 3)}=9=\|C\|
\end{aligned}
$$

Now over the weak-metric space $M_{9 \times 3}\left(\mathbb{F}_{2}\right), C$ is a $[27 ; 3 ; 7,19,25]_{2}$-code that satisfies the chain condition with

$$
\begin{aligned}
& d_{(W, 1)}=7=\left\|\operatorname{span}\left\{v_{1}\right\}\right\| \\
& d_{(W, 2)}=19=\left\|\operatorname{span}\left\{v_{1}, v_{2}\right\}\right\| \\
& d_{(W, 3)}=25=\|C\| .
\end{aligned}
$$

We observe that

$$
\operatorname{supp}(C)=\{1,4,7,11,14,17,21,24,27\}
$$

is totally ordered in weak order $W$.

As in [7], we have the monotonicity of the minimum poset-weights.

Proposition 2.1. For any $\left[n ; k ; d_{1}(C), \ldots, d_{k}(C)\right]_{q}$-code $C \leq \mathbb{F}_{q}^{n}$ we have that

$$
1 \leq d_{1}(C)<d_{2}(C)<\ldots<d_{k}(C) \leq n
$$

Proof. We first observe that $d_{r-1}(C) \leq d_{r}(C)$. Indeed, let $D_{r-1}$ and $D_{r}$ subcodes of $C$ with dimensions $r-1$ and $r$ respectively such that $\left\|D_{r-1}\right\|_{P}=$ $d_{r-1}(C)$ and $\left\|D_{r}\right\|_{P}=d_{r}(C)$. If $\left\|D_{r-1}\right\|_{P}>\left\|D_{r}\right\|_{P}$, then for any subcode 
$D_{r-1}^{\prime}<D_{r}$ of dimension $r-1$ we have that $\left\|D_{r-1}^{\prime}\right\|_{P} \leq\left\|D_{r}\right\|_{P}<\left\|D_{r-1}\right\|_{P}=$ $d_{r}(C)$. But this contradicts the minimality of $d_{r}(C)$.

We claim that the inequality $d_{r-1}(C) \leq d_{r}(C)$ is strict. Let $D$ a subcode of $C$ with dimension $r$ such that $\|D\|_{P}=d_{r}(C)$. If $i$ is a maximal element of $\operatorname{supp}(D)$, then $D_{i}:=\left\{v \in D: v_{i}=0\right\}$ is a subcode of $C$ with dimension $r-1$ such that

$$
d_{r-1}(C) \leq\left\|D_{i}\right\|_{P} \leq\|D\|_{P}-1=d_{r}(C)-1 .
$$

Since $d_{r+1}(C) \geq d_{r}(C)+1$ and $d_{k}(C) \leq n$ we immediately get the generalized Singleton bound:

Corollary 2.1. For an $\left[n ; k ; d_{1}(C), \ldots, d_{k}(C)\right]_{q}$-code $C \leq \mathbb{F}_{q}^{n}$,

$$
r \leq d_{r}(C) \leq n-k+r .
$$

Now we will show that any poset-code $C$ with $\operatorname{supp}(C)$ totally ordered satisfies the chain condition.

Theorem 2.1. Let $C$ be a code in $\mathbb{F}_{q}^{n}$, endowed with a poset-weight $w_{P}$. If $\operatorname{supp}(C)$ is a totally ordered subposet of $P$ then $C$ satisfies the chain condition.

Proof. Since $\operatorname{supp}(C)$ is totally ordered, for every $u, v \in C$ we have that either $\langle\operatorname{supp}(u)\rangle \subseteq\langle\operatorname{supp}(v)\rangle$ or $\langle\operatorname{supp}(v)\rangle \subseteq\langle\operatorname{supp}(u)\rangle$ and it follows that

$$
\|D\|_{P}=\left|\bigcup_{u \in D}\langle\operatorname{supp}(u)\rangle\right|=\max \{|\langle\operatorname{supp}(u)\rangle|: u \in D\},
$$

so that for every $j \in\{1,2, \ldots, k\}$ there is $v_{j} \in C$ such that $w_{P}\left(v_{j}\right)=d_{j}(C)$. The set $\left\{v_{1}, v_{2}, \ldots, v_{k}\right\}$ is linearly independent, since $w_{P}\left(v_{1}\right)<\ldots<w_{P}\left(v_{k}\right)$ (see Proposition 2.1) and $\operatorname{supp}(C)$ is totally ordered. Consequently

$$
\operatorname{dim}\left(\operatorname{span}\left\{v_{1}, v_{2}, \ldots, v_{j}\right\}\right)=j
$$

and

$$
\operatorname{span}\left\{v_{1}\right\}<\operatorname{span}\left\{v_{1}, v_{2}\right\}<\ldots<\operatorname{span}\left\{v_{1}, v_{2}, \ldots, v_{k}\right\}=C .
$$

Since $\left\|\operatorname{span}\left\{v_{1}, v_{2}, \ldots, v_{j}\right\}\right\|_{P}=d_{j}(C)$ for every $j \in\{1,2, \ldots, k\}$, we find that $C$ satisfies the chain condition.

Theorem 2.2. Let $C \leq \mathbb{F}_{q}^{n}$ be a code of a space endowed with a $P$-weight and suppose that $\operatorname{supp}(C)$ is a totally ordered subposet of $P$. Then there is a unique maximal flag that achieves the generalized $P$-weights hierarchy. 
Proof. Let $k=\operatorname{dim}(C),\left(d_{1}(C), d_{2}(C), \ldots, d_{k}(C)\right)$ the $P$-weights hierarchy of $C$ and $\left\{e_{1}, e_{2}, \ldots, e_{n}\right\}$ the canonical base of $\mathbb{F}_{q}^{n}$. We denote $\operatorname{supp}(C)=$ $\left\{i_{1}, i_{2}, \ldots, i_{m}\right\}$, assume it is ordered by $i_{1}<i_{2}<\ldots<i_{m}$ and let $D_{1} \leq C$ an 1-dimensional subcode of $C$ such that $\left\|D_{1}\right\|_{P}=d_{1}(C)$. We will prove that $D_{1}$ is unique. Indeed, let $D_{1}^{\prime} \leq C$ be an 1-dimensional subcode of $C$ such that $\left\|D_{1}^{\prime}\right\|_{P}=d_{1}(C)$ and $D_{1}^{\prime} \cap D_{1}=\{\mathbf{0}\}$. Then there are $u \in D_{1}$ and $v \in D_{1}^{\prime}$ such that

$$
\begin{aligned}
& u=\alpha_{1} e_{i_{1}}+\ldots+\alpha_{r-1} e_{i_{r-1}}+e_{i_{r}}, \\
& v=\beta_{1} e_{i_{1}}+\ldots+\beta_{r-1} e_{i_{r-1}}+e_{i_{r}},
\end{aligned}
$$

with $\alpha_{j} \neq \beta_{j}$ for some $j \in\{1,2, \ldots, r-1\}$ and $w_{P}(u)=w_{P}(v)=w_{P}\left(e_{i_{r}}\right)=$ $d_{1}(C)$. If

$$
l=\max \left\{j \in\{1,2, \ldots, r-1\}: \alpha_{j} \neq \beta_{j}\right\},
$$

it follow that $u-v$ is a non zero vector of $C$ such that

$$
w_{P}(u-v)=w_{P}\left(e_{i_{l}}\right)<d_{1}(C),
$$

since $l<r$. But this contradicts the minimality condition of the 1 -th $P$-weight of the code $C$, since $u-v \in C$. We conclude that $D_{1}$ is the unique subcode of $C$ that achieve the 1 -th $P$-weight of $C$.

The result follows now by induction on $\operatorname{dim}\left(D_{r}\right)=r$. Let $D_{1}<D_{2}<\ldots<$ $D_{t-1}<C$, with $t-1<k$, be the sequence of linear subspaces that achieve the $r$-th minimum Hamming $P$-weights of the code $C$ with $r \in\{1,2, \ldots, t-1\}$, assures by Theorem 2.1. Suppose that $D_{t}$ and $D_{t}^{\prime}$ are $t$-dimensional subcodes of $C$ containing $D_{t-1}$ such that $D_{t} \neq D_{t}^{\prime}$ and $\left\|D_{t}\right\|_{P}=\left\|D_{t}^{\prime}\right\|_{P}=d_{t}(C)$. Then there exist $w \in D_{t}$ and $z \in D_{t}^{\prime}$ such that

$$
\begin{gathered}
w=\gamma_{1} e_{i_{1}}+\ldots+\gamma_{s-1} e_{i_{s-1}}+e_{i_{s}}, \\
z=\eta_{1} e_{i_{1}}+\ldots+\eta_{s} e_{i_{s-1}}+e_{i_{s}},
\end{gathered}
$$

with $\gamma_{j} \neq \eta_{j}$ for some $j \in\{1,2, \ldots, s-1\}$ and $w_{P}(w)=w_{P}(z)=w_{P}\left(e_{i_{s}}\right)=$ $d_{t}(C)$. If

$$
l=\max \left\{j \in\{1,2, \ldots, s-1\}: \gamma_{j} \neq \eta_{j}\right\},
$$

then $x=w-z$ is a non zero vector of $C$ such that $w_{P}(x)=w_{P}\left(e_{i_{l}}\right)<d_{t}(C)$ and $x \notin D_{t-1}$. Then, for every linearly independent subset $\left\{y_{1}, \ldots, y_{t-1}\right\} \subset D_{t-1}$, we find that $\operatorname{span}\left\{y_{1}, \ldots, y_{t-1}, x\right\}$ is a $t$-dimensional subspace of $C$ such that $\left\|\operatorname{span}\left\{y_{1}, \ldots, y_{t-1}, x\right\}\right\|_{P} \leq d_{t}(C)-1$, contradicting the minimality of $d_{t}(C)$.

By induction, the sequence of linear subspaces $D_{1}<D_{2}<\ldots<D_{k-1}<C$ that achieve the $r$-th $P$-weights of code $C$ is unique. 
The next corollary is an immediate consequence of the previous theorem.

Corollary 2.2. Let $C$ be a linear code in $\mathbb{F}_{q}^{n}$, endowed with a posetweight $w_{P}$, such that $\operatorname{supp}(C)$ is totally ordered. If $D_{1}, D_{2}, \ldots, D_{k-1}, D_{k}=C$ is a sequence of subspaces of $C$ such that $\left\|D_{r}\right\|_{P}=d_{r}(C)$ for all $r \in\{1,2, \ldots, k\}$ and $\operatorname{dim}\left(D_{j}\right)=j$, then $D_{1}<D_{2}<\ldots<D_{k-1}<C$.

We present a lower bound for the number of codes satisfying the chain condition.

Proposition 2.2. Let $P=\{1,2, \ldots, n\}$ be a poset and suppose that $P=P_{1} \cup \ldots \cup P_{r}$ is a partition of $P$ into $r$ disjoint linear subposets. Then

$$
\sum_{i=1}^{r} \sum_{j=1}^{\nu_{i}} \prod_{k=1}^{j} \frac{\left(q^{\nu_{i}}-q^{k-1}\right)}{\left(q^{j}-q^{k-1}\right)}
$$

is a lower bound for the number of codes satisfying the chain condition in the space $\left(\mathbb{F}_{q}^{n}, d_{P}\right)$, where $\nu_{i}=\left|P_{i}\right|$ with $i \in\{1,2, \ldots, r\}$ and $r$ is larger that or equal to the size of the largest antilinear subposet of $P$.

Proof. For each subset $P^{\prime} \subseteq P$ we denoted by $\left[P^{\prime}\right]$ the subspace of $\mathbb{F}_{q}^{n}$ generated by the base $\left\{e_{i}\right\}_{i \in P^{\prime}}, e_{i}$ the canonical vector of $\mathbb{F}_{q}^{n}$. So, if $P=\bigcup_{i=1}^{r^{q}} P_{j}$ is a partition (disjoint union of sets) we have that $\mathbb{F}_{q}^{n}$ is a direct sum

$$
\left[P_{1}\right] \oplus \ldots \oplus\left[P_{r}\right] .
$$

As $P_{i}$ is a linear subposet for every $i \in\{1,2, \ldots, r\}$, Theorem 2.1 ensures that any code $C \leq\left[P_{i}\right]$ satisfies the chain condition. Therefore the number of $j$-dimensional codes of $\left[P_{i}\right]$ satisfying the chain condition equals

$$
\prod_{k=1}^{j} \frac{\left(q^{\nu_{i}}-q^{k-1}\right)}{\left(q^{j}-q^{k-1}\right)}
$$

for each $i \in\{1,2, \ldots, r\}$. This completes the proof of the Proposition. The Dilworth's Theorem (see [4]) assures that $P$ can be partitioned into $r$ chains if the largest antilinear in the poset $P$ has size $r$.

Let $\left\{e_{1}, e_{2}, \ldots, e_{n}\right\}$ be the canonical base of $\mathbb{F}_{q}^{n}$. The trivial code $C=$ $\operatorname{span}\left\{e_{1}, e_{2}, \ldots, e_{n}\right\}$ obviously satisfies the chain condition, independently of the poset structure on $\mathbb{F}_{q}^{n}$. This fact can be generalized in the following way: let

$$
C=C_{1} \oplus C_{2} \oplus \ldots \oplus C_{t}
$$


be a linear code in $\mathbb{F}_{q}^{n}$, endowed with a $P$-weight $w_{P}$, such that

$$
\operatorname{supp}(C)=\operatorname{supp}\left(C_{1}\right) \cup \operatorname{supp}\left(C_{2}\right) \cup \ldots \cup \operatorname{supp}\left(C_{t}\right)
$$

is a disjoint union of chains. Its follows from theorem 2.2 there is a unique chain

$$
D_{1}^{(i)} \subset D_{2}^{(i)} \subset \ldots \subset D_{k_{i}}^{(i)}=C_{i}
$$

such that $\left\|D_{j}^{(i)}\right\|_{P}=d_{j}\left(C_{i}\right)$ for every $j \in\left\{1,2, \ldots, k_{i}\right\}$ for all $i \in\{1,2, \ldots, t\}$. Suppose now that

$$
\left\|D_{k_{i}}^{(i)}\right\|_{P} \leq\left\|D_{1}^{(i+1)}\right\|_{P}
$$

for every $i \in\{1,2, \ldots, t-1\}$. Then, using the same kind of reasoning used in the proof of Theorem 2.1, it is easy to see that $C$ satisfies the chain condition. Moreover, there is a unique chain that realizes the hierarchy of the generalized $P$-weights, as in Theorem 2.2:

$$
D_{(0,1)} \subset \ldots \subset D_{\left(0, k_{1}\right)} \subset \ldots \subset D_{(t-1,1)} \subset \ldots \subset D_{\left(t-1, k_{t}\right)}
$$

with

$$
D_{(i, j)}=D_{j}^{(i+1)} \oplus D_{k_{i}}^{(i)} \oplus D_{k_{i-1}}^{(i-1)} \oplus \ldots \oplus D_{k_{0}}^{(0)}
$$

and $D_{r_{0}}^{(0)}=\{\mathbf{0}\}$

\section{References}

[1] R. Brualdi, J. S. Graves, M. Lawrence, Codes with a poset metric, Discrete Math., 147 (1995), 57-72, http://dx.doi.org/10.1016/0012-365X(94)00228B.

[2] J. Y. Hyun, H. K. Kim, The poset structures admitting the extended binary Hamming code to be a perfect code, Discrete Math., 288 (2004), 37-47, http://dx.doi.org/10.1016/j.disc.2004.07.010.

[3] F. J. MacWilliams, N. J. A. Sloane, The theory of error-correcting codes, North-Holland Mathematical Library (1997).

[4] B. S. W. Schroder, Ordered Sets - An Introduction, Birkhauser (2003).

[5] M. M. Skriganov, Coding theory and uniform distributions, St. Petersburg Math. J., 13 (2002), 301-337. 
[6] M. Yu Rosenbloom, M. A. Tsfasman, Codes for the m-metric, Probl. Inf. Transm., 33 (1997), 45-52.

[7] V. K. Wei, Generalized Hamming weights for linear code, IEEE Trans. Inform. Theory, 37 (1991), 1412-1418, http://dx.doi.org/10.1109/18.133259.

[8] V. K. Wei, K. Yang, On the generalized Hamming weights for product code, IEEE Trans. Inform. Theory, 39 (1993), 1709-1713, http://dx.doi.org/10.1109/18.259662. 
